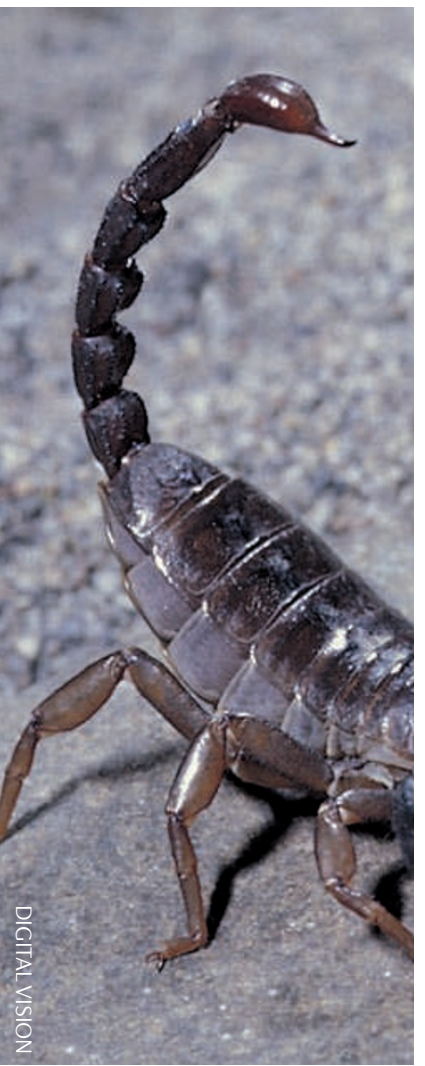

RNA PROCESSING

\title{
Viral infection has a sting in the tail
}

Viruses have an armoury of mechanisms to manipulate host gene expression and divert cellular resources to viral replication. A recent report finds that the weapon of choice for one herpesvirus is a system that increases host mRNA turnover by altering polyadenylation. This viral mechanism could lead to a re-examination of the conventional view of how the poly(A) tail affects mRNA stability in eukaryotes.

During its lytic cycle, Kaposi's sarcoma-associated herpesvirus destroys the host transcriptome by massive degradation of mRNA. Although this process - host shutoff - is known to be mediated by the viral factor SOX (shutoff and exonuclease), the molecular basis of mRNA destruction has remained enigmatic.

Lee and Glaunsinger showed that wild type SOX - but not mutant
SOX, which causes a defect in RNA turnover - induces aberrant lengthening of poly(A) tails by poly(A) polymerase II. To find out how SOX mediates this hyperadenylation and subsequent mRNA degradation, they monitored the subcellular localization of poly(A) binding proteins (PABPs) in the presence and absence of SOX and tested the effects of PABP knockdown on host shutoff. SOX expression caused cytoplasmic PABP (PABPC) to relocalize to the nucleus. PABPC was found to be necessary for mRNA destruction, and nuclear PABP (PABPN) was necessary for aberrant tail lengthening. As these PABPs are involved in the normal regulation of poly(A) length and in maintaining mRNA stability, the authors propose that these proteins are subverted by viral infection to upregulate mRNA turnover by an as yet unidentified RNase.

Although poly(A) tails in prokaryotes are known to recruit exonucleases, the traditional view of polyadenylation in eukaryotes is that it stabilizes transcripts. So the discovery that it can also act as a signal for degradation is particularly surprising. Therefore, as well as identifying a novel mechanism by which viruses manipulate their hosts, this report suggests that the influences of polyadenylation on eukaryotic RNA processing are more diverse than previously supposed.

Mary Muers

ORIGINAL RESEARCH PAPER Lee, Y. J. \& Glaunsinger, B. A. Aberrant herpesvirus-induced polyadenylation correlates with cellular messenger RNA destruction. PLoS Biol. 7 , e1000107 (2009) 